\title{
Optimized In Vivo Detection of Dopamine Release Using ${ }^{18}$ F-Fallypride PET
}

\author{
Jenny Ceccarini ${ }^{1}$, Elske Vrieze ${ }^{2}$, Michel Koole ${ }^{1}$, Tom Muylle ${ }^{1}$, Guy Bormans ${ }^{3}$, Stephan Claes $^{2}$, and Koen Van Laere ${ }^{1}$ \\ ${ }^{1}$ Division of Nuclear Medicine, University Hospital and Katholieke Universiteit, Leuven, Belgium; ${ }^{2}$ Department of Psychiatry, \\ University Hospital, Leuven, Belgium; and ${ }^{3}$ Laboratory for Radiopharmacy, Katholieke Universiteit, Leuven, Belgium
}

The high-affinity $D_{2 / 3}$ PET radioligand ${ }^{18} \mathrm{~F}$-fallypride offers the possibility of measuring both striatal and extrastriatal dopamine release during activation paradigms. When a single ${ }^{18} \mathrm{~F}$-fallypride scanning protocol is used, task timing is critical to the ability to explore both striatal and extrastriatal dopamine release simultaneously. We evaluated the sensitivity and optimal timing of task administration for a single ${ }^{18} \mathrm{~F}$-fallypride PET protocol and the linearized simplified reference region kinetic model in detecting both striatal and extrastriatal reward-induced dopamine release, using human and simulation studies. Methods: Ten healthy volunteers underwent a single-bolus ${ }^{18} \mathrm{~F}$-fallypride PET protocol. A reward responsiveness learning task was initiated at $100 \mathrm{~min}$ after injection. PET data were analyzed using the linearized simplified reference region model, which accounts for time-dependent changes in ${ }^{18} \mathrm{~F}$-fallypride displacement. Voxel-based statistical maps, reflecting task-induced $\mathrm{D}_{2 / 3}$ ligand displacement, and volume-of-interest-based analysis were performed to localize areas with increased ligand displacement after task initiation, thought to be proportional to changes in endogenous dopamine release ( $\gamma$ parameter). Simulated time-activity curves for baseline and hypothetical dopamine release functions (different peak heights of dopamine and task timings) were generated using the enhanced receptor-binding kinetic model to investigate $\gamma$ as a function of these parameters. Results: The reward task induced increased ligand displacement in extrastriatal regions of the reward circuit, including the medial orbitofrontal cortex, ventromedial prefrontal cortex, and dorsal anterior cingulate cortex. For task timing of $100 \mathrm{~min}$, ligand displacement was found for the striatum only when peak height of dopamine was greater than $240 \mathrm{nM}$, whereas for frontal regions, $\gamma$ was always positive for all task timings and peak heights of dopamine. Simulation results for a peak height of dopamine of $200 \mathrm{nM}$ showed that an effect of striatal ligand displacement could be detected only when task timing was greater than $120 \mathrm{~min}$. Conclusion: The prefrontal and anterior cingulate cortices are involved in reward responsiveness that can be measured using ${ }^{18} \mathrm{~F}$-fallypride PET in a single scanning session. To measure both striatal and extrastriatal dopamine release, the height of dopamine released and task timing need to be considered in designing activation studies depending on regional $D_{2 / 3}$ density.

Received Oct. 25, 2011; revision accepted May 23, 2012.

For correspondence or reprints contact: Jenny Ceccarini, Division of Nuclear Medicine, University Hospital Leuven-Gasthuisberg, Herestraat 49, 3000 Leuven, Belgium.

E-mail: jenny.ceccarini@uz.kuleuven.be

Published online Aug. 29, 2012.

COPYRIGHT (C) 2012 by the Society of Nuclear Medicine and Molecular Imaging, Inc.
Key Words: extrastriatal dopamine release; ${ }^{18} \mathrm{~F}$-fallypride; reward task; LSSRM; task timing

J Nucl Med 2012; 53:1565-1572

DOI: 10.2967/jnumed.111.099416

$\mathbf{T}$ he mesolimbic pathway, where dopaminergic neurons project from the ventral tegmental area to the nucleus accumbens, has been viewed as the core neuronal circuitry involved in reward processing (1). Reward capacity serves to elicit approach and consummatory behaviors, maintains and prevents their extinction, and induces subjective feelings of pleasure, including reinforcement and incentive (2).

Multiple sources of evidence have shown alterations in mesolimbic dopaminergic neurotransmission when reward functions are impaired (3). Until now, most PET experiments on dopamine $D_{2 / 3}$ neurotransmission have used the specific dopamine benzamide antagonist ${ }^{11} \mathrm{C}$-raclopride (4). Because of its low affinity and hence limited capacity to measure extrastriatal $\mathrm{D}_{2 / 3}$ receptors, the role of extrastriatal dopamine release in the mechanism of responses to reinforcing stimuli has received much less attention. Such a role has, however, been suggested by various studies showing that the reward value, the expected reward value (5), and other reinforcers also have representations in the orbitofrontal cortex (6) and in the anterior cingulate cortex (7). Furthermore, reward expectation and reward prediction errors are attributed to prefrontal and cingulate dopaminergic signaling (8).

With the development of high-affinity radioligands for the $\mathrm{D}_{2 / 3}$ receptor such as ${ }^{11} \mathrm{C}$-FLB-457, ${ }^{18} \mathrm{~F}$-desmethoxyfallypride, and especially ${ }^{18} \mathrm{~F}$-fallypride, it has become possible to noninvasively quantify extrastriatal $\mathrm{D}_{2 / 3}$ receptor densities during pharmacologic (9) and nonpharmacologic paradigms $(10-12)$. Even in regions such as the cortex and thalamus, where $D_{2 / 3}$ receptor densities are one or two orders of magnitude lower than in the striatum, it is possible to measure changes in nondisplaceable binding potential, $\mathrm{BP}_{\mathrm{ND}}(13)$.

However, the relatively slow kinetics of ${ }^{18} \mathrm{~F}$-fallypride and the difference in receptor density and, hence, uptake and washout kinetics in various brain regions (e.g., slower in striatum than in cortex) led to the observation that when 
a single scanning session is used, task timing is critical for assessing striatal and extrastriatal dopamine release simultaneously. The influence of task timing on the kinetics of the ligand has not systematically been studied on the basis of actual observed parameters and might be considered a real challenge (11).

The aim of this study was to evaluate the sensitivity of a single ${ }^{18} \mathrm{~F}$-fallypride PET protocol and kinetic linearized simplified reference region modeling (LSSRM) (14) based on observations in experimental designs such as reward task performance correlated to self-reported measures of anhedonia (15).

Through simulation studies, we quantitatively estimated the ability of LSSRM to detect dopamine release simultaneously in both extrastriatal and striatal regions by analyzing the kinetic characteristics of ${ }^{18} \mathrm{~F}$-fallypride with variable heights of dopamine released and variable timings of task initiation.

\section{MATERIALS AND METHODS}

\section{Participants}

Ten healthy right-handed, medication-free female volunteers (mean age $\pm \mathrm{SD}, 33.3 \pm 8.2 \mathrm{y}$ ) were enrolled in the human imaging study. In light of sex differences in dopamine release (16), in this study only female volunteers were included. Additionally, we chose female subjects because we are currently using the same ${ }^{18}$ F-fallypride protocol in a depression study in which a female preponderance is expected. All participants were examined to exclude current or past neuropsychiatric diseases, especially mood disorders and substance addiction. All subjects underwent blood and urine analysis and fasted for at least $4 \mathrm{~h}$ before undergoing PET. Informed consent was obtained from all participants before the investigations. The study was approved by the local ethics committee and was performed according to the latest World Medical Association Declaration of Helsinki.

\section{Reward Task}

The task that was used to elicit dopamine release was a validated computerized signal-detection reward-based paradigm involving monetary gains $(15,17)$. The reward task required identifying - by pressing a button-2 difficult-to-discriminate visual stimuli, a long $(13 \mathrm{~mm})$ or short $(11.5 \mathrm{~mm})$ mouth, displayed for $100 \mathrm{~ms}$ on a cartoon face on a computer screen. During the task, 6 blocks of 100 trials each were presented, and within each block the 2 stimuli were displayed with equal frequency. In each block, volunteers immediately received a monetary reward after approximately 40 correct answers. The task is described in more detail in the supplemental material (available online at http://jnm.snmjournals.org).

\section{Imaging Procedures}

Tracer preparation is described in the supplemental material. Before receiving the ${ }^{18} \mathrm{~F}$-fallypride injection, the volunteers were placed with the head restrained using a vacuum cushion to minimize movement during the PET acquisition.

Subjects received, on average, $179 \pm 17 \mathrm{MBq}$ of ${ }^{18} \mathrm{~F}$-fallypride in a slow intravenous 10-s bolus injection. A PET dynamic emission was initiated simultaneously on injection and was acquired in 3-dimensional mode on an HR + scanner (Siemens). Data were acquired in 60-s frames during the first 6 min and in 120-s frames thereafter. The PET emission was acquired in 2 blocks, following a previously reported one-day PET protocol $(11,12)$. The first block, with a duration of $64 \mathrm{~min}$, represented baseline ${ }^{18} \mathrm{~F}$-fallypride kinetics. After a short break, the second emission dataset was collected for another $70 \mathrm{~min}$, in which the first $20 \mathrm{~min}$ represented an extension of the baseline scan and the last 50 min represented scanning after initiation of the reward task (at $100 \mathrm{~min}$ after injection).

Images were reconstructed using a standard 3-dimensional filtered backprojection algorithm, including scatter and measured attenuation correction $\left({ }^{68} \mathrm{Ge}\right.$ source). A volumetric T1-weighted and standard transverse T2-weighted brain MRI scan was obtained from each volunteer to exclude structural brain abnormalities and for coregistration purposes (1.5-T Vision scanner; Siemens).

\section{Data Processing and Kinetic Model}

For each subject, the dynamic reconstructed images were realigned using a rigid transformation to correct for potential effects of head movement and then coregistered to the corresponding MRI scan. All individual image data were then spatially normalized to a specific T1-weighted template in Montreal Neurologic Institute space. These calculations were done using SPM2 (Statistical Parametric Mapping; Wellcome Trust Centre for Neuroimaging). The normalized images were smoothed with a 3-dimensional gaussian filter (4 $\mathrm{mm}$ in full width at half maximum) (11). For each subject, 2 binary masks were defined on the corresponding normalized MRI scan, using an in-house-created set of volumes of interest, defined according to Brodmann areas on the basis of the Talairach atlas and constructed using the PMOD software volume-of-interest tool (PMOD Inc.); one mask was created to include all cerebral regions to be analyzed, and a second mask was defined on the cerebellum.

Kinetic parameters were estimated by LSSRM (10-12,14), implemented in-house in Matlab (MathWorks Inc). LSSRM accounts for time-dependent changes in radiotracer binding, influx, and clearance induced by cognitive or drug effects in a single scan session, with the inclusion of a baseline and an activation condition. LSSRM assumes that the physiologic steady state is not maintained throughout the paradigm but allows the dissociation rate of ligand $k_{2 \mathrm{a}}\left(k_{2 \mathrm{a}}=k_{2} /\left[1+\mathrm{BP}_{\mathrm{ND}}\right]\right)$, where $k_{2}$ is the tissueto-plasma efflux constant in the tissue region, to change over time in response to local fluctuations in dopamine concentration. A detailed description of the functional equations describing LSSRM and defining the parameters is given in the supplemental material. The dopamine-radioligand competition at the receptor sites is reflected by a temporal change of $k_{2 \mathrm{a}}$, which is accounted for by a time-dependent parameter $k_{2 \mathrm{a}}+\gamma \cdot \mathrm{h}(\mathrm{t})$, where $\gamma$ represents the amplitude of the ligand displacement and the function $\mathrm{h}(\mathrm{t})(\mathrm{h}(\mathrm{t})=$ $\exp [-\tau(\mathrm{t}-\mathrm{T})])$ describes the rapid change after task onset and dissipation over time ( $\tau$ controls the rate at which activation effects die away and $\mathrm{T}$ indicates the timing of task initiation [100 min]). The residual sum of the squares was examined for different $\tau$-values $(0.02-0.05)$ and was minimal for $\tau=0.03 \mathrm{~min}^{-1}$. The same value was used in previous publications $(11,12)$. An increased $k_{2 \mathrm{a}}$ therefore reflects a decreased $\mathrm{BP}_{\mathrm{ND}}$ for $\mathrm{D}_{2 / 3}$ receptors, as can be ascribed to an increased dopamine release, which will result in a positive value of $\gamma$. The cerebellum was used as the reference region because of the negligible density of dopamine $\mathrm{D}_{2 / 3}$ receptors in this region (18).

For each subject, quantitative parametric maps of $\mathrm{R}\left(=K_{1} / K_{1 \mathrm{r}}\right.$ [reference region]), $k_{2}, k_{2 \mathrm{a}}, \mathrm{BP}_{\mathrm{ND}}$, and $\gamma$ were computed. A volume-of-interest analysis was additionally performed by estimating the parameters $\mathrm{R}, K_{2}, K_{2 \mathrm{a}}, \mathrm{BP}_{\mathrm{ND}}$, and $\gamma$ using LSSRM 
and the PET time-activity curves over regions displaying prominent dopamine release.

\section{Statistics}

LSSRM uses weighted linear least squares analysis for parameter estimation, providing an estimate of the covariance matrix of the parameters. Using this estimate, statistical significance was assessed computing individual statistical voxelwise $t$ maps of the $\gamma$ parameter $(t=\gamma / \operatorname{SD}(\gamma)$, where $\operatorname{SD}(\gamma)$ is the $\operatorname{SD}$ parametric value for $\gamma$ ). This $t$ statistic was then used to determine whether the time-varying parameter, $\gamma$, yielded a significant improvement in the model (i.e., rejection of the null hypothesis), similar to the method described by Alpert et al. (14). Based on the degrees of freedom (11), a threshold of $t>5$ was used, which corresponds to a $P$ value of less than 0.000005 (1-tailed) or a Bonferronicorrected $P$ value of less than 0.05 (0.05/average total number of voxels analyzed per subject $[=11,000])$. Next, the spatial extent of the estimated task-induced dopamine release was presented as the percentage of voxels exceeding a threshold within each volume of interest, similar to prior studies (11).

\section{Simulation Studies}

To corroborate our results obtained with the current experimental design and to estimate quantitatively the ability of LSSRM to detect dopamine release simultaneously in both extrastriatal and striatal regions, we analyzed the kinetic characteristics of ${ }^{18} \mathrm{~F}$-fallypride with variable peak heights of dopamine released and variable task timings through simulation studies, starting from the experimental observed parameters.

Because dopamine release highly depends on the dynamics of free radioligand in the tissue when the task is initiated, finding the optimum baseline scan duration reflecting on task timing may increase the ability of LSSRM to detect dopamine release simultaneously in regions with low (extrastriatal) and high (striatal) $\mathrm{D}_{2 / 3}$ concentration $(11,19)$. To investigate the influence of task timing on LSSRM results, we first generated noiseless simulations of ${ }^{18} \mathrm{~F}$-fallypride time-activity curves under baseline and stimulus conditions (created with dopamine release functions, i.e., dopamine perturbations).

The simulations were performed using an extension of the standard compartmental model commonly used in tracer kinetic PET analysis $(20,21)$. The enhanced receptor-binding model considers both the time-varying fluctuating levels of the dopamine concentration and its competition with the radiotracer for the available $\mathrm{D}_{2 / 3}$ binding sites.

This method allowed us to investigate directly the process of dopamine elevation, including time and amplitude information, within the activation detection studies. The plasma input function of tracer concentration was modeled as a biexponential decay function. Time-activity curve simulations of the dopamine perturbations were created by fixing the necessary kinetic constants of ${ }^{18} \mathrm{~F}$-fallypride, $\mathrm{B}_{\max }$ (the total number of $\mathrm{D}_{2 / 3}$ receptor sites), and dopamine binding kinetics for the $\mathrm{D}_{2 / 3}$ receptor to realistic values obtained from previous kinetic analyses. The rate constants of ${ }^{18} \mathrm{~F}$-fallypride were based on Christian et al. (22): for striatum we used $K_{1}=0.17 \mathrm{~mL} / \mathrm{min} / \mathrm{g}, k_{2}=0.2 \mathrm{~min}^{-1}, k_{\mathrm{on}}=0.04(\mathrm{pmol} / \mathrm{mL})$ $(\mathrm{min})^{-1}, k_{\text {off }}=0.043 \mathrm{~min}^{-1}$, and $B^{\prime}{ }_{\max }=54 \mathrm{pmol} / \mathrm{mL}$; for the frontal cortex we used $K_{1}=0.21 \mathrm{~mL} / \mathrm{min} / \mathrm{g}, k_{2}=0.24 \mathrm{~min}^{-1}$, $k_{\text {on }}=0.22(\mathrm{pmol} / \mathrm{mL})(\mathrm{min})^{-1}, k_{\text {off }}=0.043 \mathrm{~min}^{-1}$, and $B^{\prime}{ }_{\text {max }}=$ $0.3 \mathrm{pmol} / \mathrm{mL}$. The association and dissociation rate constants for endogenous dopamine at the $D_{2 / 3}$ receptors were fixed as $0.25(\mathrm{pmol} / \mathrm{mL})(\mathrm{min})^{-1}$ and $25 \mathrm{~min}^{-1}$, respectively (23). By as- suming that $\mathrm{B}_{\max }$ was $54 \mathrm{pmol} / \mathrm{mL}$ and that $50 \%$ of total receptors are occupied by endogenous dopamine at steady state, we implicitly assumed that $\mathrm{B}^{\prime}{ }_{\text {max }}$ was $27 \mathrm{pmol} / \mathrm{mL}$ (the available receptor concentration at steady state), in agreement with the parameter estimates by Christian et al. (22). Kinetic parameters were quantitatively close to the parameter values determined by the human ${ }^{18} \mathrm{~F}$-fallypride time-activity curves obtained from the experimental results.

To simulate ${ }^{18} \mathrm{~F}$-fallypride stimulus time-activity curves, hypothetical dopamine release functions $\left(\mathrm{F}^{\mathrm{DA}}\right)$ were generated according to Morris et al. (20):

$$
\mathrm{F}^{\mathrm{DA}}(\mathrm{t})=\mathrm{DA}^{\text {basal }}+\mathrm{G}(\mathrm{t}-\mathrm{T})^{\alpha} \exp [-\beta(\mathrm{t}-\mathrm{T})] ; \mathrm{t} \geq \mathrm{T} .
$$

$\mathrm{DA}^{\text {basal }}$ is the baseline dopamine concentration (set at $100 \mathrm{nM}$ (23)), $\mathrm{G}$ is a leading coefficient, and $\alpha$ and $\beta$ control the change of dopamine level, and $\mathrm{T}$ is the timing of task initiation. We have assumed that the time delay of the onset of the function $(20,24)$ was zero, thus hypothesizing that the peak height of dopamine perturbation would occur at a peak time of $\alpha / \beta$. Because for a specific stimulus condition the appropriate corresponding dopamine level is not known, different values of $\mathrm{G}$ were used to determine the sensitivity to a particular endogenous concentration. The following settings were used to generate dopamine perturbation curves that peaked at the same time but differed with respect to $\mathrm{G}$, and hence different peak heights of dopamine: $\alpha=2.7, \beta=0.4$, and $\mathrm{G}=0.008,0.025,0.042$, $0.058,0.076,0.092$, and 0.109 . This choice of $\alpha$ and $\beta$ was obtained from simulations to achieve a peak height of dopamine of 120$370 \mathrm{nM}$, which represents values up to 4 times the baseline dopamine concentration-within the expected values for cognitive and reward processes (23). The observed time-activity curve effects in our experimental reward task were within this simulated range of peak heights of dopamine (160-200 nM). Moreover, each dopamine perturbation curve was simulated with defined task timings ranging from 80 to $220 \mathrm{~min}$ with a 10 -min step. To apply our experimental design to the simulation paradigm, for each task timing dopamine perturbation was simulated each $3 \mathrm{~s}$ (approximately the duration of a single trial) for $50 \mathrm{~min}$. In this way, similar timing of task-induced dopamine perturbations was hypothetically obtained.

Each simulated condition was evaluated by quantifying the estimated $\gamma$-value and the maximal percentage difference between stimulus time-activity curves and baseline time-activity curve at different peak heights of dopamine and task timings.

Simulated time-activity curves were constituted at 1-min intervals and were implemented using a simulator previously developed for the kinetic competition model (25).

Second, to better approximate the real experimental setting, additional simulations of noisy data were also computed. Simulations of noisy data were computed by adding gaussian random noise with SD proportional to $\mathrm{C}_{\mathrm{m}} \cdot \mathrm{e}^{\lambda \mathrm{t}} / d \mathrm{t}$, similar to the noise model described by Alpert et al. (14). The proportionality factor with the real data $\mathrm{C}_{\mathrm{m}}$ was obtained from the ${ }^{18} \mathrm{~F}$-fallypride human scans and estimated over the last $20 \mathrm{~min}$ of the acquisition, and $\lambda$ denotes the decay constant for ${ }^{18} \mathrm{~F}$. All parameters of the model were also estimated taking into account noise.

\section{RESULTS}

\section{Reward-Induced Extrastriatal Dopamine Release}

Globally, the reward task produced the intended behavioral effects because participants developed a response bias in favor of the more frequently rewarded rich stimulus (15). 
Figure 1 shows a representative example of voxel-based parametric images of the kinetic parameters. LSSRM was directly used to fit all voxels within the binary mask, estimating parametric images for $k_{2}, k_{2 \mathrm{a}}, \mathrm{R}$, and $\gamma$. In addition, the $\mathrm{BP}_{\mathrm{ND}}$ image (calculated as $\left.\mathrm{BP}_{\mathrm{ND}}=\left[\left(k_{2} / k_{2 \mathrm{a}}\right)-1\right]\right)$, the covariance image $(\operatorname{SD}(\gamma))$, and the statistic $t$ map for $\gamma$ were generated for each subject.

The reward task induced a significant bilateral decrease in ${ }^{18} \mathrm{~F}$-fallypride binding in the medial orbitofrontal cortex, ventromedial prefrontal cortex, and dorsal anterior cingulate cortex (Fig. 2). The location of the maximum dopamine release is given in stereotactic Talairach coordinates, which are $[x, y, z]=[-2,37,-25](t=6.4)$ for the medial orbitofrontal cortex, $[x, y, z]=[-11,66,6](t=5.6)$ for the ventromedial prefrontal cortex, and $[x, y, z]=[-2,49,13]$ $(t=5.1)$ for the dorsal anterior cingulate cortex.

Table 1 shows the individual size (in percentage) of significant $(t>5)$ voxels in these activated areas. For all regions, no significant difference between the left and the right hemispheres was observed.

Dynamic time-activity curves were then extracted over the extrastriatal regions showing significantly increased radioligand displacement and fitted with the LSSRM, giving estimates for the kinetic parameter. Figure 3A shows an example of dynamic time-activity curve fit, which was generated from a volume of interest covering the left medial orbitofrontal cortex of subject 7. Compared with other subjects, this participant had the largest measured effect in this region. As can be seen from this figure, the LSSRM fit shows a marked decrease in ${ }^{18} \mathrm{~F}$-fallypride concentration soon after the task was initiated, indicating prompt task-induced dopamine release. Normalized residuals for fitting the data with

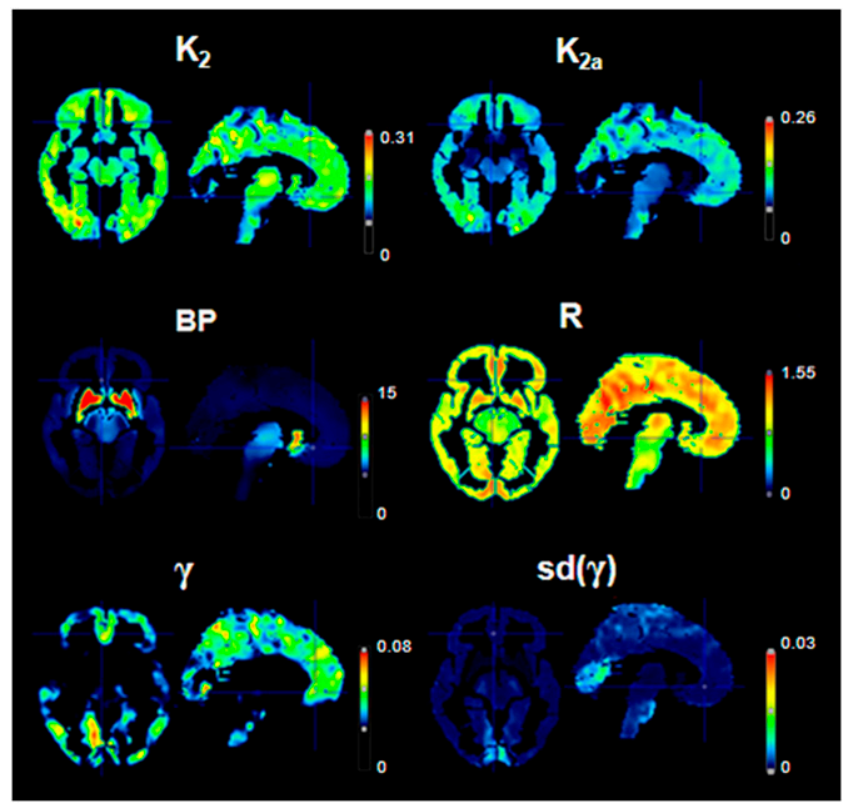

FIGURE 1. Example of $k_{2}, k_{2 \mathrm{a}}, \mathrm{BP}_{\mathrm{ND}}, \mathrm{R}, \gamma$, and $\mathrm{SD}(\gamma)$ parametric images calculated by LSSRM for subject 9 . For each parametric map, respective color bar is shown on right side.

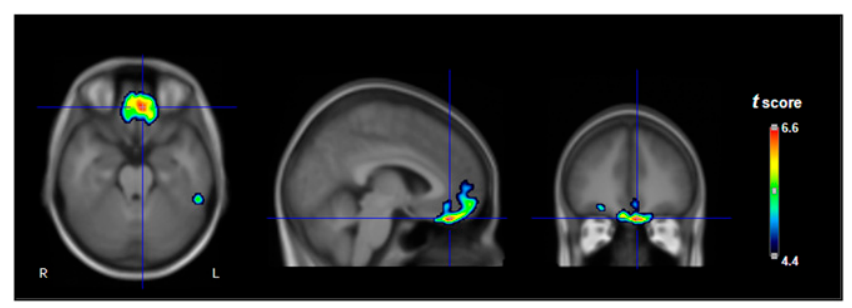

FIGURE 2. Average statistical parametric $t$ map of $\gamma$ in transverse, coronal, and sagittal sections overlaid on T1-weighted MRI template, showing significant bilateral reward-induced ${ }^{18} \mathrm{~F}$-fallypride displacement in medial orbitofrontal cortex, ventromedial prefrontal cortex, and dorsal anterior cingulate cortex.

and without $\gamma$ are plotted in Figure 3B, demonstrating the improvement in the quality of the LSSRM fit when the "activation" $\gamma$-term was included. For each activated region, the estimates for the kinetic parameters are given in Table 2.

As described elsewhere in full detail, the extent of taskinduced dopamine release was also correlated with reward task performance and self-reported measures of anhedonia (15).

\section{Simulations of Dopamine Release}

Influence of Task Timing and Dopamine Level on $\gamma$-Estimation. Figure 4 presents the simulation results of the $\gamma$-estimates for the striatum (Fig. 4A) and the frontal cortex (Fig. 4B), based on ${ }^{18} \mathrm{~F}$-fallypride time-activity curves obtained applying dopamine perturbations characterized by increasing peak heights of dopamine (from 120 to $370 \mathrm{nM}$ ) and increasing task timings (from 80 to $220 \mathrm{~min}$ ). These results clearly show that both peak height of dopamine and task timing have a high impact on the value of $\gamma$. When considering $\gamma$-values for task timing of $100 \mathrm{~min}$ (as in the experimental protocol), positive $\gamma$-values are obtained for the putamen only when peak height of dopamine exceeds $245 \mathrm{nM}$ (Fig. 4A), whereas for the frontal cortex, $\gamma$ is always positive for all peak heights of dopamine and all task timings

\section{TABLE 1}

Task-Induced Dopamine Release Presented by Percentage of Voxels Exceeding Threshold of $t>5$ Within Each Activated Region

\begin{tabular}{|c|c|c|c|c|c|c|}
\hline 1 & $46 \%$ & $56 \%$ & $18 \%$ & $10 \%$ & $11 \%$ & $8 \%$ \\
\hline 2 & $29 \%$ & $21 \%$ & $16 \%$ & $9 \%$ & $6 \%$ & $9 \%$ \\
\hline 3 & $8 \%$ & $7 \%$ & $30 \%$ & $14 \%$ & $20 \%$ & $35 \%$ \\
\hline 4 & $8 \%$ & $4 \%$ & $7 \%$ & $16 \%$ & $4 \%$ & $21 \%$ \\
\hline 5 & $12 \%$ & $9 \%$ & $23 \%$ & $18 \%$ & $20 \%$ & $8 \%$ \\
\hline 6 & $57 \%$ & $50 \%$ & $26 \%$ & $29 \%$ & $22 \%$ & $10 \%$ \\
\hline 7 & $70 \%$ & $64 \%$ & $30 \%$ & $35 \%$ & $30 \%$ & $20 \%$ \\
\hline 8 & $37 \%$ & $41 \%$ & $12 \%$ & $24 \%$ & $31 \%$ & $20 \%$ \\
\hline 9 & $26 \%$ & $17 \%$ & $66 \%$ & $72 \%$ & $90 \%$ & $88 \%$ \\
\hline 10 & $8 \%$ & $12 \%$ & $41 \%$ & $23 \%$ & $48 \%$ & $30 \%$ \\
\hline
\end{tabular}

BA11 = Brodmann area 11 (medial orbitofrontal cortex); BA10 = Brodmann area 10 (ventromedial prefrontal cortex); $\mathrm{BA} 32=$ Brodmann area 32 (dorsal anterior cingulate cortex). 


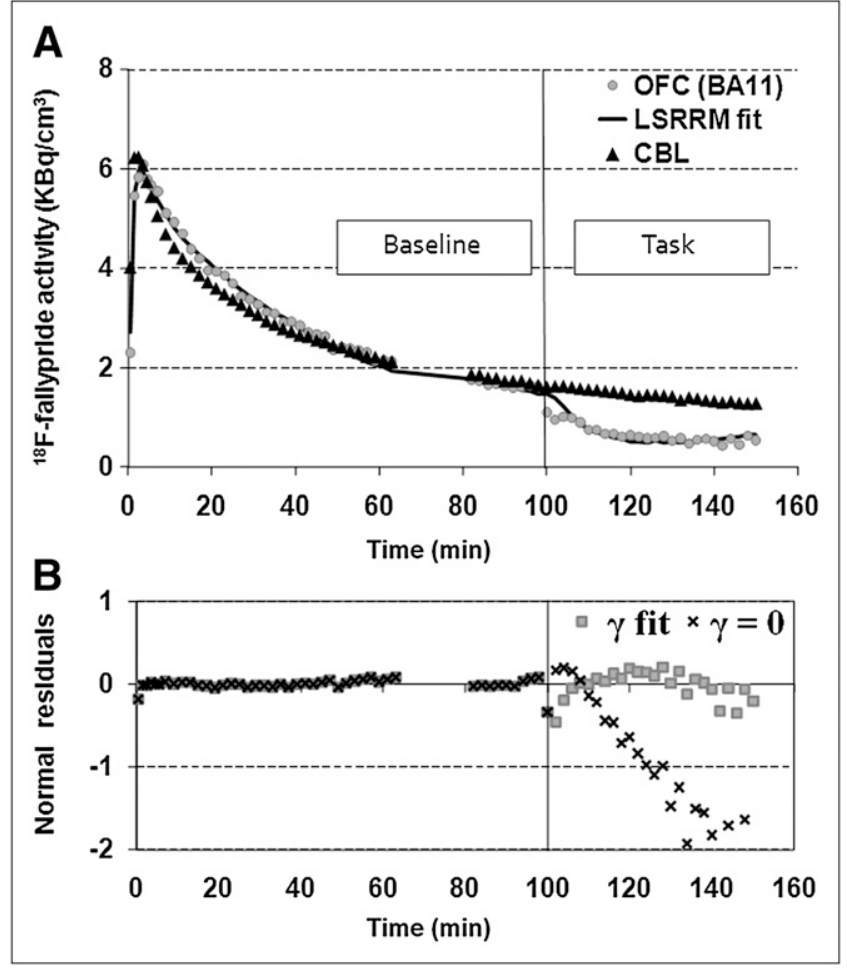

FIGURE 3. Example of ${ }^{18} \mathrm{~F}$-fallypride displacement after task initiation ( $T=100 \mathrm{~min}$, vertical line) in activated left medial orbitofrontal cortex (OFC) for subject 7 ( $t=8.25$ ), compared with cerebellum (CBL) (A). Improvement of LSSRM fit to data including $\gamma(B)$.

(Fig. 4B). Thus, the sensitivity of the protocol may have been suboptimal to detect low or modest changes in dopamine release in the striatum, later activation time points may be needed for this region, and the protocol was more optimized for the frontal cortex.

Influence of Dopamine Level on Striatal ${ }^{18}$ F-Fallypride Binding. Figure 5 shows the effect of different peak heights of dopamine on the simulated ${ }^{18} \mathrm{~F}$-fallypride activities in the striatum. Figure 5A displays hypothesized dopamine perturbation curves beginning at a time point of 100 min during the simulated scans and proceeding for the following $50 \mathrm{~min}$. Peak height of dopamine ranged from 120 to $370 \mathrm{nM}$, and since all dopamine perturbation curves were characterized by the same temporal parameters, each dopamine perturbation curve peaked at the same time.

The effect of these dopamine perturbation curves on the striatal ${ }^{18} \mathrm{~F}$-fallypride time-activity curve is shown in Figure 5B. Figure 5C shows the plot of the maximal percentage difference between the predicted baseline and stimulus time-activity curve during the activation condition versus increasing peak height of dopamine. For peak heights of dopamine below $120 \mathrm{nM}$, the difference is nearly zero since the baseline dopamine concentration was set at $100 \mathrm{nM}$. This near-linear dependence suggests that the effect of activation on the ${ }^{18} \mathrm{~F}$-fallypride time-activity curves, and thus dopamine detectability, is higher if the peak height of dopamine is higher.

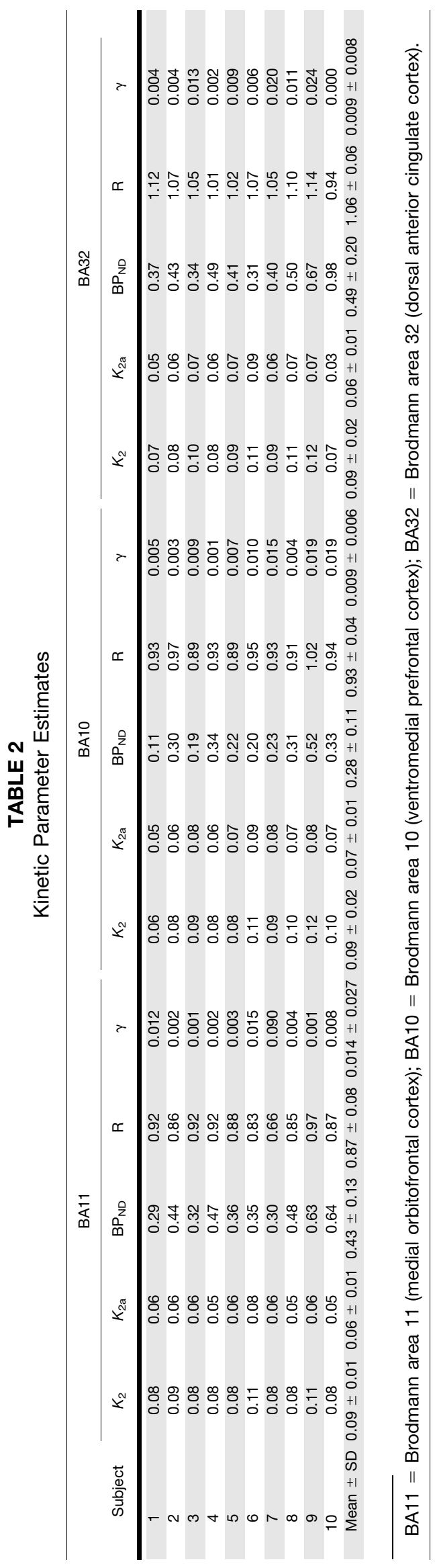

Optimized Detection of Dopamine Release - Ceccarini et al. 


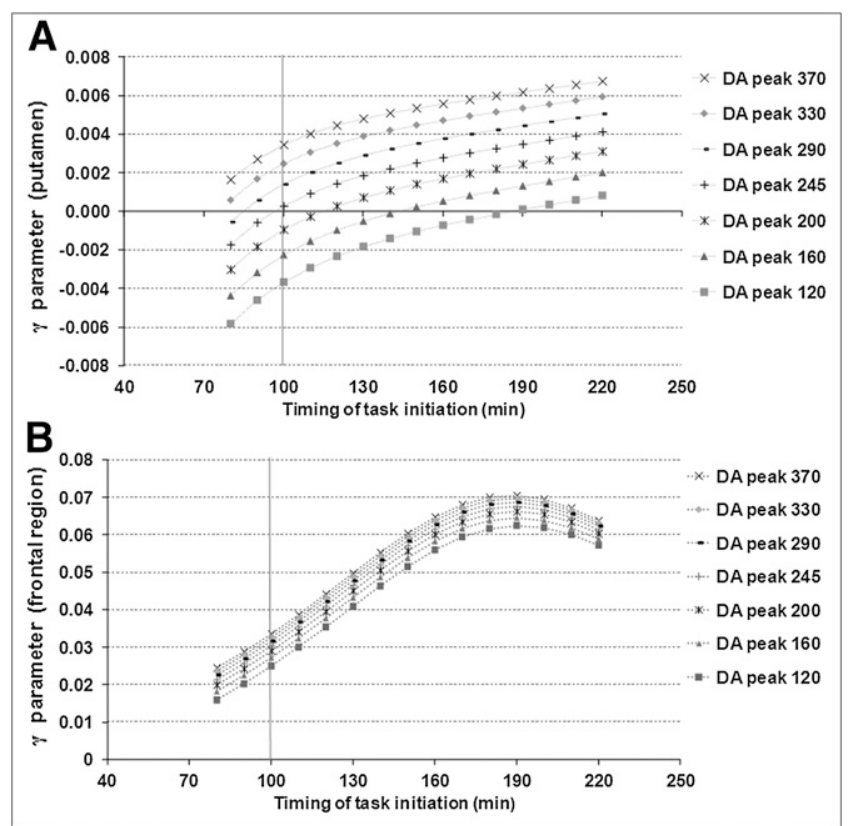

FIGURE 4. Evolution of $\gamma$ parameter over time corresponding to different peak heights of dopamine and task timings simulated for putamen (A) and for frontal region (B). Vertical line at $T=100 \mathrm{~min}$ indicates task initiation of experimental protocol.

Influence of Added Noise on the Simulations. Figure 6A shows an example of the LSSRM fit to noisy baseline timeactivity curve data and stimulus time-activity curve data for a peak height of dopamine of $245 \mathrm{nM}$ induced by a task beginning at $190 \mathrm{~min}$. The simulation analysis revealed that the uncertainty intervals of the simulated $\gamma$ parameter (SD $(\gamma)$ ) were not significantly dependent on task timing (Fig. 6B), albeit with slightly higher variability for lower peak heights of dopamine. For the lowest peak height of dopamine $(120 \mathrm{nM})$, the difference between $\gamma$-estimates obtained with noisy and noise-free data was $2.0 \% \pm 3.4 \%$, whereas for the highest peak height of dopamine $(370 \mathrm{nM})$, the difference was only $0.5 \% \pm 0.5 \%$ (Fig. 6C). Therefore, even when one is considering realistic noise levels with a stimulus application at 190-220 min, there would be only a small bias for the estimated $\gamma$-values. Therefore, these simulations with noisy data similar in amplitude to that observed by experiment demonstrate that noise does not represent a possible confounding factor or bias for the findings.

\section{DISCUSSION}

Extending the research on the reward circuit that until recently focused on striatal changes, we explored in vivo extrastriatal endogenous dopamine release in healthy humans while they were performing a validated learning reward task. We found that ${ }^{18} \mathrm{~F}$-fallypride can be successfully displaced from extrastriatal $D_{2 / 3}$ receptors in detectable amounts even if these are low-binding regions, supporting the hypothesis that prefrontal and cingulate regions are critically involved in reward processing. Immediately after task initiation, ${ }^{18} \mathrm{~F}$-fallypride binding significantly decreased in the medial orbitofrontal cortex, ventromedial prefrontal cortex, and dorsal anterior cingulate cortex. The $\mathrm{BP}_{\mathrm{ND}}$ values estimated using LSSRM are supported by the $\mathrm{D}_{2 / 3}$ binding profiles in the frontal regions as found in previous studies, which reported that $\mathrm{BP}_{\mathrm{ND}}$ ranged between 0.2 and $0.4(26)$.

These results are consistent with current neurocognitive models that implicate these regions as important network components in reward processing. Neurons in the primate orbitofrontal cortex have shown 3 forms of reward-related activity during the performance of delayed response tasks (27). Also, goals and decision values have been correlated to orbitofrontal cortex activity using a decision-making paradigm (28). The dorsal anterior cingulate cortex has been implicated in a variety of higher cognitive processes, such as detecting and predicting errors and monitoring conflicting behavioral responses. Single-unit recording studies suggest a relevant role of the anterior cingulate cortex in actionreward combinations and goal-based action selection $(8)$. The ventromedial prefrontal cortex is implicated in the learning of contingencies based on the outcome of a rewarding sit-
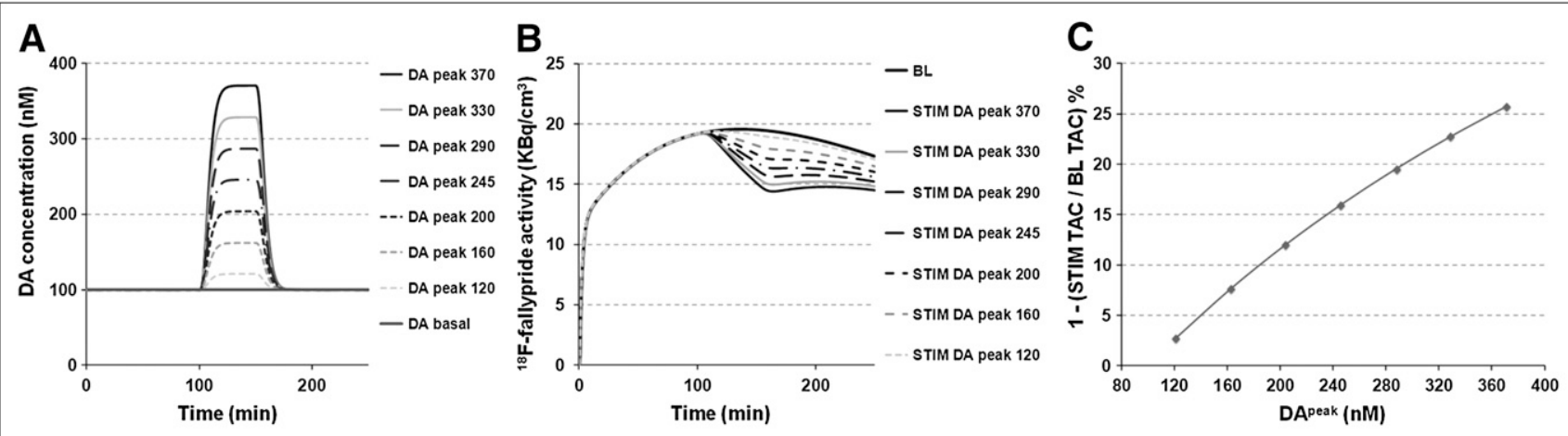

FIGURE 5. Hypothesized dopamine perturbations with identical dopamine peak times used in simulated ${ }^{18} \mathrm{~F}$-fallypride stimulus timeactivity curve and different peak heights of dopamine (A). Horizontal line at $100 \mathrm{nM}$ indicates baseline dopamine concentration. For each dopamine perturbation shown in A, B shows baseline time-activity curve and stimulus time-activity curves and C shows maximal percentage differences between baseline time-activity curve and stimulus time-activity curves during activation condition in putamen. $\mathrm{BL}=\mathrm{baseline;}$ STIM = stimulus. 


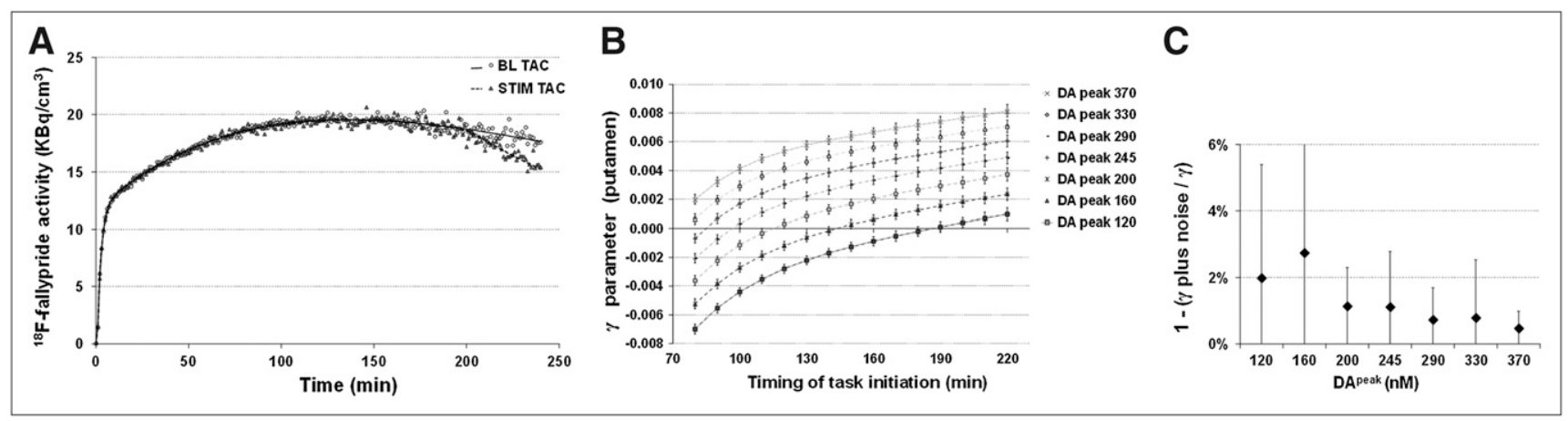

FIGURE 6. (A) Effect of noise on ${ }^{18} \mathrm{~F}$-fallypride kinetics. Solid line is fit to noisy baseline time-activity curve data, and dotted line is fit to noisy stimulus time-activity curve data obtained for task timing of $190 \mathrm{~min}$ and peak dopamine height of $245 \mathrm{nM}$. (B) Evolution of $\gamma$ parameter estimates over time corresponding to different peak heights of dopamine and task timings simulated for putamen, obtained with noisy and noise-free data. Error bars indicate SD. (C) Percentage differences between $\gamma$-estimates obtained with noisy ( $\gamma$ plus noise) and noise-free $(\gamma)$ data for different peak heights of dopamine. $\mathrm{BL}=$ baseline; STIM = stimulus.

uation. It has been observed that the ventromedial prefrontal cortex becomes more active by presentation of reward outcomes than by presentation of nonreward outcomes (29) and that the ventromedial prefrontal cortex receives information based on the expectancy of reinforcement, which is used for adaptive decision making (5).

Because it is known that both prefrontal and striatal regions may be involved in mediating the response to rewarding stimuli, we also investigated whether a single ${ }^{18} \mathrm{~F}$-fallypride session and corresponding kinetic modeling analysis would be able to detect both extrastriatal and striatal changes in dopamine levels. Using a kinetic competition model, we investigated the dependency of the $\gamma$ parameter in the LSSRM on timing of task initiation and dopamine release levels.

First, we found that the possible assessment of an increase in ligand displacement in response to dopamine release in the task condition could be significantly enhanced through delay of task initiation. When the lowest peak height of dopamine is considered, simulations indeed show that the predicted effect of striatal dopamine release could be detected if the activation task were to start around 190$200 \mathrm{~min}$ after injection. Contrary to frontal regions with a low $\mathrm{D}_{2 / 3}$ concentration, for which we estimated positive $\gamma$-values for all peak heights of dopamine, the detectability of increased rate of ligand displacement for regions with high binding thus highly depends on the task-induced peak height of dopamine. These findings may explain the lack of detection of striatal dopamine release when the task was initiated $100 \mathrm{~min}$ after injection. This would mean that the task-induced peak height of dopamine is certainly lower than $240 \mathrm{nM}$, as is consistent with previous suggestions (23).

Such a finding is in accordance with ${ }^{18} \mathrm{~F}$-fallypride kinetic behavior, which shows a marked effect of $D_{2 / 3}$ receptor concentration on the equilibrium time of the radioligand and hence on the corresponding $\mathrm{BP}_{\mathrm{ND}}$ estimation $(19,30)$. It takes longer to reach equilibrium in the $\mathrm{D}_{2 / 3}$ receptor-rich regions and therefore to obtain a reliable estimate for $\mathrm{BP}_{\mathrm{ND}}$.
From our simulations (Fig. 5B) and in accordance with Christian et al. (30), a task activation time point of at least $120 \mathrm{~min}$ is needed for the $\mathrm{D}_{2 / 3}$-rich regions corresponding to a peak height of dopamine of $200 \mathrm{nM}$, whereas the extrastriatal regions with a significantly lower $\mathrm{D}_{2 / 3}$ concentration equilibrate much more rapidly. Therefore, if the dopamine perturbation is applied early during the neurotransmitterradioligand competition, the ${ }^{18} \mathrm{~F}$-fallypride occupancy would not be at the equilibrium situation in $\mathrm{D}_{2 / 3}$-rich regions yet, thus reflecting an underestimation of binding change. On the other hand, if the activation paradigm is applied at a later time point once the plateau is reached, more time would be available to achieve the equilibrium before the stimulus application, and subsequently LSSRM might ensure parameter stability and reliability.

When the effect of peak height of dopamine on the difference between stimulus and baseline time-activity curves was examined, a positive near-linear correlation was observed between the maximal stimulus and baseline timeactivity curve difference and dopamine release. It has been shown that such a linear correlation, which could also be described by a change in $\mathrm{BP}_{\mathrm{ND}}$, appears to be confined to a narrow range of dopamine release (24). Nevertheless, because the $15 \%-25 \%$ difference between simulated time-activity curves for peak height of dopamine ranged from 245 to $370 \mathrm{nM}$, it is reasonable to conclude that striatal endogenous dopamine activation could be detected.

Some limitations require consideration. First, the results of our simulations have to be interpreted in light of the assumptions we made, such as for the time course and shape of the dopamine response patterns. We assumed that changes in model parameters occurred immediately after activation was initiated. Second, using LSSRM, we assumed not only that the activation effect diminished with time but also that the decrease in effect was exponential. Alternatively, models such as the recent lp-ntPET model, a basis function augmentation of the LSSRM method (31), could be used, because the lp-ntPET technique also permits temporal characterization of neurotransmitter fluctuations, including estimation 
of the response onset, peak time, and sharpness. Therefore, lp-ntPET simulations might further increase sensitivity for estimation of neurotransmitter dynamics from PET data. Moreover, although LSSRM has several advantages by virtue of its requiring only single-day scanning, the model implies that time-dependent alterations in regional cerebral blood flow may not be fully accounted for. However, as shown by previous simulation studies $(11,14)$, it is unlikely that regional cerebral blood flow-related changes would add major perturbations in ligand displacement studies using cognitive and reward activation paradigms.

\section{CONCLUSION}

The prefrontal and anterior cingulate cortices are involved in reward responsiveness shown by dopamine release in vivo. Furthermore, on the basis of simulation studies performed over a range of timings and heights of released dopamine, we have shown that a single ${ }^{18} \mathrm{~F}$-fallypride imaging protocol and LSSRM analysis can be used for simultaneous measurement of extrastriatal and striatal dopamine release. Improvements in the experimental design, such as a postponement of task initiation, should increase the relative detection sensitivity of striatal dopamine release, and 120 190 min after injection are needed to evaluate both.

\section{DISCLOSURE STATEMENT}

The costs of publication of this article were defrayed in part by the payment of page charges. Therefore, and solely to indicate this fact, this article is hereby marked "advertisement" in accordance with 18 USC section 1734.

\section{ACKNOWLEDGMENTS}

We acknowledge the PET radiopharmacy for their skilled collaboration. Koen Van Laere and Stephan Claes are both senior clinical investigators of the Flemish Fund of Scientific Research (FWO). No other potential conflict of interest relevant to this article was reported.

\section{REFERENCES}

1. Schultz W. Dopamine neurons and their role in reward mechanisms. Curr Opin Neurobiol. 1997;7:191-197.

2. Robbins TW, Everitt BJ. Neurobehavioural mechanisms of reward and motivation. Curr Opin Neurobiol. 1996;6:228-236.

3. Nestler EJ, Carlezon WA Jr. The mesolimbic dopamine reward circuit in depression. Biol Psychiatry. 2006;59:1151-1159.

4. Egerton A, Mehta MA, Montgomery AJ, et al. The dopaminergic basis of human behaviors: a review of molecular imaging studies. Neurosci Biobehav Rev. 2009;33:1109-1132.

5. Gottfried JA, O’Doherty J, Dolan RJ. Encoding predictive reward value in human amygdala and orbitofrontal cortex. Science. 2003;301:1104-1107.

6. Kringelbach ML. The human orbitofrontal cortex: linking reward to hedonic experience. Nat Rev Neurosci. 2005;6:691-702.

7. Amiez C, Joseph JP, Procyk E. Reward encoding in the monkey anterior cingulate cortex. Cereb Cortex. 2006;16:1040-1055.
8. Rushworth MF, Behrens TE. Choice, uncertainty and value in prefrontal and cingulate cortex. Nat Neurosci. 2008;11:389-397.

9. Riccardi P, Li R, Ansari MS, et al. Amphetamine-induced displacement of $\left[{ }^{18} \mathrm{~F}\right]$ fallypride in striatum and extrastriatal regions in humans. Neuropsychopharmacology. 2006;31:1016-1026.

10. Badgaiyan RD, Fischman AJ, Alpert NM. Dopamine release during human emotional processing. Neuroimage. 2009;47:2041-2045.

11. Christian BT, Lehrer DS, Shi B, et al. Measuring dopamine neuromodulation in the thalamus: using [F-18]fallypride PET to study dopamine release during a spatial attention task. Neuroimage. 2006;31:139-152.

12. Lataster J, Ceccarini J, Collip D, et al. Psychosocial stress is associated with in vivo dopamine release in human ventromedial prefrontal cortex: a positron emission tomography study using $\left[{ }^{18}\right.$ F]fallypride. Neuroimage. 2011;58:1081-1089.

13. Innis RB, Cunningham VJ, Delforge J, et al. Consensus nomenclature for in vivo imaging of reversibly binding radioligands. J Cereb Blood Flow Metab. 2007;27:1533-1539.

14. Alpert NM, Badgaiyan RD, Livni E, Fischman AJ. A novel method for noninvasive detection of neuromodulatory changes in specific neurotransmitter systems. Neuroimage. 2003;19:1049-1060.

15. Vrieze E, Ceccarini J, Pizzagalli DA, et al. Measuring extrastriatal dopamine release during a reward learning task. Hum Brain Mapp. November 23, 2011 [Epub ahead of print].

16. Riccardi P, Zald D, Li R, et al. Sex differences in amphetamine-induced displacement of $\left[{ }^{18} \mathrm{~F}\right]$ fallypride in striatal and extrastriatal regions: a PET study. Am J Psychiatry. 2006;163:1639-1641.

17. Pizzagalli DA, Jahn AL, O'Shea JP. Toward an objective characterization of an anhedonic phenotype: a signal-detection approach. Biol Psychiatry. 2005;57:319-327.

18. Lammertsma AA, Hume SP. Simplified reference tissue model for PET receptor studies. Neuroimage. 1996;4:153-158.

19. Vernaleken I, Peters L, Raptis M, et al. The applicability of SRTM in $\left[{ }^{18} \mathrm{~F}\right]$ fallypride PET investigations: impact of scan durations. J Cereb Blood Flow Metab. 2011;31:1958-1966.

20. Morris ED, Yoder KK, Wang C, et al. ntPET: a new application of PET imaging for characterizing the kinetics of endogenous neurotransmitter release. Mol Imaging. 2005;4:473-489.

21. Morris ED, Fisher RE, Alpert NM, Rauch SL, Fischman AJ. In vivo imaging of neuromodulation using positron emission tomography: optimal ligand characteristics and task length for detection of activation. Hum Brain Mapp. 1995;3:35-55.

22. Christian BT, Narayanan T, Shi B, Morris ED, Mantil J, Mukherjee J. Measuring the in vivo binding parameters of $\left[{ }^{18} \mathrm{~F}\right]$-fallypride in monkeys using a PET multipleinjection protocol. J Cereb Blood Flow Metab. 2004;24:309-322.

23. Fisher R, Morris ED, Alpert NM, Fischman AJ. In vivo imaging of neuromodulatory synaptic transmission using PET: a review of relevant physiology. Hum Brain Mapp. 1995;3:24-34.

24. Yoder KK, Wang C, Morris ED. Change in binding potential as a quantitative index of neurotransmitter release is highly sensitive to relative timing and kinetics of the tracer and the endogenous ligand. J Nucl Med. 2004;45:903-911.

25. Muylle T, Dupont P, Van Laere K. On the detection of endogenous ligand release with PET: a simulation study [abstract]. Neuroimage. 2008;(suppl 2):T75.

26. Mukherjee J, Christian BT, Dunigan KA, et al. Brain imaging of ${ }^{18} \mathrm{~F}$-fallypride in normal volunteers: blood analysis, distribution, test-retest studies, and preliminary assessment of sensitivity to aging effects on dopamine D-2/D-3 receptors. Synapse. 2002;46:170-188.

27. Schultz W, Tremblay L, Hollerman JR. Reward processing in primate orbitofrontal cortex and basal ganglia. Cereb Cortex. 2000;10:272-284.

28. Hare TA, O'Doherty J, Camerer CF, Schultz W, Rangel A. Dissociating the role of the orbitofrontal cortex and the striatum in the computation of goal values and prediction errors. J Neurosci. 2008;28:5623-5630.

29. Knutson B, Fong GW, Adams CM, Varner JL, Hommer D. Dissociation of reward anticipation and outcome with event-related fMRI. Neuroreport. 2001;12:3683-3687.

30. Christian BT, Narayanan TK, Shi B, Mukherjee J. Quantitation of striatal and extrastriatal D-2 dopamine receptors using PET imaging of $\left[{ }^{18} \mathrm{~F}\right]$ fallypride in nonhuman primates. Synapse. 2000;38:71-79.

31. Normandin MD, Schiffer WK, Morris ED. A linear model for estimation of neurotransmitter response profiles from dynamic PET data. Neuroimage. 2012; 59:2689-2699. 